\title{
Integration of Wearable Smart Sensor for Improving e-Healthcare
}

\author{
Vijey Thayananthan and Abdullah Basuhail \\ Department of Computer Science \\ King Abdulaziz University \\ Jeddah 21589, Saudi Arabia
}

\begin{abstract}
Analyzing health conditions using sensors is one of the daily activities in a healthcare organization. The purpose of this research is to improve the e-healthcare formulated through the integration of wearable smart sensors and miniaturized devices. In this research, monitoring glucose level of the diabetic is considered as an example of the non-linear problem in which we show that accuracy and efficiency of e-healthcare can be achieved through Multiple-input, multiple-output (MIMO) system. In this novel technique, Pn-manifolds, which are the nonlinear mathematical approach, provide the flexible rate and enhance the accuracy and efficiency of the medical systems in the e-healthcare services.
\end{abstract}

Keywords-Smart sensors; miniaturized devices; e-healthcare applications; MIMO; manifolds

\section{INTRODUCTION}

Sensors and miniaturized devices enhance the e-healthcare facilities in many ways. Since they have started to collect the information, healthcare units use them in either inside or outside of the human body. In most of the e-healthcare application, smart sensors which are the advanced version of the absorbing (receiving), storing and transmitting device or machine, can be utilized in many different cases such as monitoring patients' continuous fitness. Thus, we studied the integration of wearable sensors for monitoring patients [1]. A comprehensive understanding of the MIMO wireless network provides us for establishing the wireless body area network, and the way in which information of the wrist, knee, and ankle could be used to improve patient care via wearable sensors [2]. When the efficient framework is available at home, healthcare facilities for elders will be manageable quickly [3]. Despite remote monitoring, e-healthcare helps healthcare units to avoid all complex problems and provides the best performance of services.

Miniaturized medical devices based on nanotechnology and DNA (deoxyribonucleic acid) computers provide implantable facilities. In e-healthcare application, miniaturization is a future innovation technology because it allows healthcare units to use the integration of wearable sensors. Further, medical devices with smaller, superior features and capabilities, are a revolution for motivating the next generation of the e-healthcare system.

Our contributions which focus on e-healthcare applications based on the miniaturized devices, communication network and integration of wearable sensors are summarized below.
A theoretical model of a healthcare system based on MIMO wireless channel which is the extension and advanced version of the basic wireless channels. Here, integration of wearable smart sensor (IWSS) plays an important role to reach the maximum e-healthcare facilities and benefits.

We have focused on the enhancements of benefits such as accuracy obtained from the selected example which is the popular e-health application (diabetes) over MIMO network and IWSS. According to [4]-[8], people who have diabetes of Type 1 and 2 take-ups a number of recovery actions such as medications. Despite all possible actions, diabetic are suffering from serious attacks created by diabetes. Here, patients may wear an acquired jacket which transfers all the symptoms through the integrated sensors. The theoretical model demonstrates the proposed scheme is highly efficient when we use the novel approach which deals with MIMO scheme.

In this research, the overall performance of e-healthcare application involved with MIMO communication is considered. Minimizing errors during the medical tests or examination procedures will improve the accuracy and efficiency of the e-healthcare technology. Efficient design and analysis allow us to minimize the errors of the e-healthcare applications when the proposed technique, which uses the novel approach of MIMO channel with feedback and Pnmanifold is employed in medical communication [9]. Here, the Pn-manifold influenced with the channel matrix is used for calculating best resolution and quantization, which provides the high quality communication channel and flexible rates to the e-healthcare system.

The rest of the paper is organized as follows. Section II focuses on literature review and background. In Section III, we provide details of sensors used in e-healthcare. Section IV explains the proposed model and brief methodology of the proposed scheme. Theoretical analysis of the e-healthcare services obtained from the selected e-health application (diabetes) is organized in Section V. In Section VI, overall conclusions are written based on the theoretical analysis and results.

\section{LITERATURE REVIEW AND BACKGROUND}

Regarding the e-healthcare applications, we have extensively studied the uses of the Pn-manifold [9] and how it has been designed to improve the accuracy, rate and connectivity performance of the wireless communication systems. The MIMO system needs Pn-manifold because it 
reduces the complexity and power consumptions. This research depends on quick response time known as the warning time that should save the patient's life. Therefore, Pn-manifold is considered as a novel approach and chosen to increase the response time. Further, it provides the better resolution and spectrum which can be achievable using the rank concept of Pn-manifold matrix and its computation.

According to [10], applications of the e_healthcare system depend on the efficient system architecture which includes latest key technologies, Internet of things and big health system influenced with human-cloud integration [11], [12]. Design and performance analysis of wireless body sensor network allows patients to improve the e_healthcare problems at different levels through android [13]. There are many wearable sensors such as Neurosky, ABM-B-Alert, Quasar, NeuroelectricsEnobio, SmartCap Nia2, Melon, Zeo and Emotiv-epocneuroheadset as described in [14].

Continuous glucose monitoring is also dependent on the diabetic's activities which include the physical exercises and diet controls during the day or specific time [15]. Healthcare units provide latest monitoring facilities, but continuous online solutions and treatment depend on the advice of the medical professionals and level of the glucose.

A wearable UHF RFID-based EEG system allows patients in the healthcare units to keep all the mobile facilities which can be adaptable [16]. Further, some valid healthcare solutions [17] are proposed for developing a mobile healthcare system that includes the network devices and machines. Despite the Wireless Body LAN network for health monitoring, uses of the sensors between the implanted device and external devices have been integrated. According to [18], wearable electroencephalogram systems not only identify the heart problems but also monitor all the heart-related symptoms and transfer the e-healthcare units.

Using real-time and secure health monitoring systems, patients get all the facilities quickly and securely [19]. A full framework is proposed for a remote brain-machine interface (RMBI) system that uses a wearable headband with dry electrodes EEG nano-sensors with Bluetooth functionality [20], [21]. Further, RBMI system helps elderly people to improve their basic skills in the e_healthcare applications. Here, healthcare unit uses EEG signals acquired through tethered and portable sensor systems.

According to [22], remote monitoring of e-healthcare services such as patients' health and personal records can be handled through the IWSS. Hospitals in a remote area may not have that many interactions which not only increases the unnecessary costs but also reduces the accuracy of the performance related to the patient's monitoring systems. Remote monitoring of elderly people's fitness and other ehealthcare services can be analyzed through the virtual MIMObased wireless communication [23]. Mobile hospitals are also growing in western countries, and they reduce the costs and time.

Everywhere, uses of devices such as phones increase the facilities of the e-healthcare services such as healthcare information and administrations. As shown in Fig. 1, uses of e- healthcare sensors (devices) in cities, remote areas and others (space and underwater) are varied with types of cares, which allow us to calculate the overall cost per treatment.

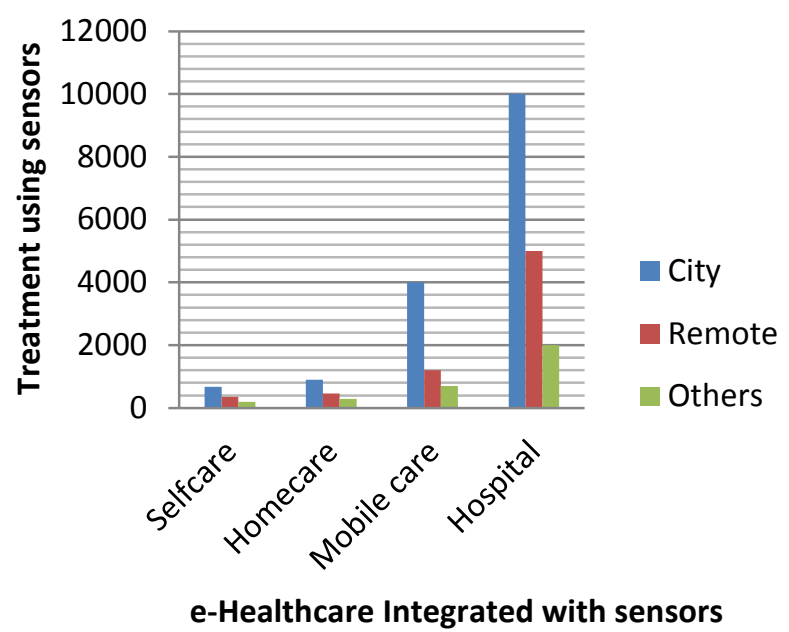

Fig. 1. Estimate treatments/day using wearable system integrated with sensors.

According to the recent news, e-healthcare systems have been practiced for a decade, but still, they are growing with wearable smart sensor facilities. New technologies are also open many directions to implement the e-healthcare applications in the latest style with multiple environments. Despite the IWSS in medical communication, e-healthcare is not only used for clinical applications but also it is implemented in other medical administrations. Based on [24], Content-Centric Network (CCN) helps not only to improve the e-healthcare, but it also allows us to develop a framework over $5 \mathrm{G}$ which is the primary enabler for e-healthcare applications. Further, it solves many existing problems such as the traffic, packet loss, etc. Since this CCN router provides the caching capability, proposed framework has become popular in all services used in e-healthcare applications. Although mobile Internet developers are the main customers for e-healthcare services, m-health solutions using bio-monitoring sensor should be added to the mobile healthcare environments [25]. In mobile healthcare, integration of wearable smart sensor in medical communication will navigate our body with all facilities while we are on the move.

\section{SENSORS IN ELECTRONIC HEALTHCARE}

In e-healthcare applications, sensors have been widely used in many aspects such as analyzing temperature. Healthcare technology uses the tiny machines as sensors which take maximum roles in all aspects of the medical and healthcare systems. It helps everybody from basic inquiry level to the final operation or surgery stage through medical communication. Currently, key challenges focusing on communication technology are growing in e-healthcare applications. Since healthcare facilities have modern approaches, medical professionals utilize the miniaturization of medical equipment used in the organs that are not functioning properly. Also, other challenges such as efficiency of surgery through online and patient safety can be implemented through 
IWSS. There are plenty of other challenges in medical history because diseases will never end and change to different form.

\section{A. Smart Sensors}

Sensors consist of absorbing elements such as photoelectric, ultrasonic and some chemical substances which release the absorbed reaction when the e-healthcare application is present. Some sensors consist of impedance such as inductive and capacitive. In IWSS, a number of smart sensors collect necessary medical information and exchange data related to healthcare in all environments. Both smart and conventional sensors simply take the reactions and symptoms of the patients' conditions which could be physical, biological or chemical input and convert them to the measured value in a digital format.

Smart sensors are a combination of both small memory and physical connection which is able to communicate with the signal processor and data network [26]. They produce the electrical output when the electronic circuit is combined with interfacing. Smart sensors are upgraded version of the traditional sensors embedded with current technology which includes the Bio and Nano processing, SDN switching, etc. Flexible and reliable smart sensors are required in order to observe the energy which depends on the density of blood. Although there are many types of sensors used widely for many applications, IWSS improves the healthcare technology. Further, IWSS provides the quick decision which allows healthcare units to deliver the correct actions to all patients. Thus, smart sensors are required to measure and maintain the up to date healthcare information.

\section{B. Biosensors}

In medical applications, scientists use biosensors which observe all characteristics of human organs' actions and reactions. It can be integrated with modern technologies which are powerful to speed the procedures. Diabetes symptoms of the blood samples should have more glucose molecules within the fixed volume of the blood vessels. Biosensors integrated with smart sensors, and other medical devices can provide natural facilities to collect a lot of information related to glucose molecules during the molecular communication [27]. Molecular communication is already invented and verified with text messages received through the biosensors used within the specific organ.

\section{Sensors in Wireless Networks}

Based on the current e_healthcare applications and wireless network, we all believe that sensors are the most important to take the quick decisions. Current e-health applications depend on the wireless networks such as WLAN, WiFi, WiMax. The sensors integrated with the wireless network are also available in a healthcare environment where wireless sensors network (WSN) have been applied most of the e-health applications. Wearable smart sensor integrated within medical communication systems used in e-healthcare applications can be categorized into three different forms; observing and testing equipment, controlling units and communication systems. In this research, IWSS involved with miniaturized communication devices (MCD) is used for internal and external data interfacing. The internal data interfacing unit must be MCD because it should be within or around the specific organ. The external data interfacing unit may be MCD or other existing devices which depend on the healthcare situation and environments. Instead of using wearable sensors, the MCD can be designed using smart sensors intelligently [28]. Although MCD has many intelligent approaches in the e-healthcare applications, the goal is that the miniaturized sensor is able to be engaged in multiple environmental conditions which allow the healthcare units to monitor and note multiple measurements [29].

A basic MIMO configuration has $\mathrm{N} \geqslant 2$ transmitters and $M \geqslant 2$ receivers, which use the feedback link with manifolds considered with evidence of non-linear phenomena. Further, body-worn distributed MIMO system, which is introduced in [30] which provides wireless LAN (WLAN) capabilities for improving e-healthcare applications. In this distributed MIMO system, MIMO configuration has $\mathrm{N}=2$ and $\mathrm{M}=3$ antennas. Appropriate MIMO configurations and their transmission capabilities over multiple channels are essential to improve the e-healthcare facilities. Wireless healthcare system based on MIMO allows healthcare units to reduce the interferences when body area network integrated with sensors is employed in e-healthcare [31]. Despite the advantages of MIMO, accuracy, and optimization of monitoring all medical tests in e-healthcare applications depend on the novel design of the theoretical model. Here, the greatest testing advantage is that MIMO system uses the efficient design which focuses on feedback and Pn-manifold. Further, it provides high quality e-healthcare technologies with low-cost because it uses less energy consumption depending on better spectrum, and resolution. Comparing a legacy system of healthcare monitoring, mobile IWSS needs efficient signal detection depended on MIMO when multiple problems related to e-healthcare are analyzed with some form of priority such as an emergency.

\section{Proposed TeChNiQue}

As shown in Fig. 2, we have proposed a theoretical model as a novel technique which provides the accurate measurement of Type 2 diabetes (non-insulin-dependent diabetes) when we test the glucose level of Type 2 diabetic.

\section{A. Theoretical Model}

As a method, a theoretical model which enables us to analyze the e-healthcare problems through the appropriate MIMO communication channel is proposed. This novel approach supports us to verify the medical communication capabilities and allows us to evaluate the performance of the ehealthcare facilities. In this theoretical model, a non-linear mathematical approach based on Pn-manifold plays an important role to improve the communication facilities of the e-healthcare system.

$$
\frac{k_{B} T}{2}
$$




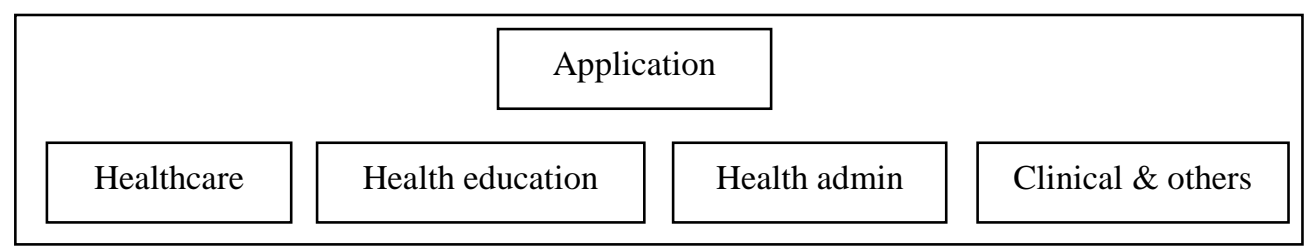

Infrastructure with interfacing unit
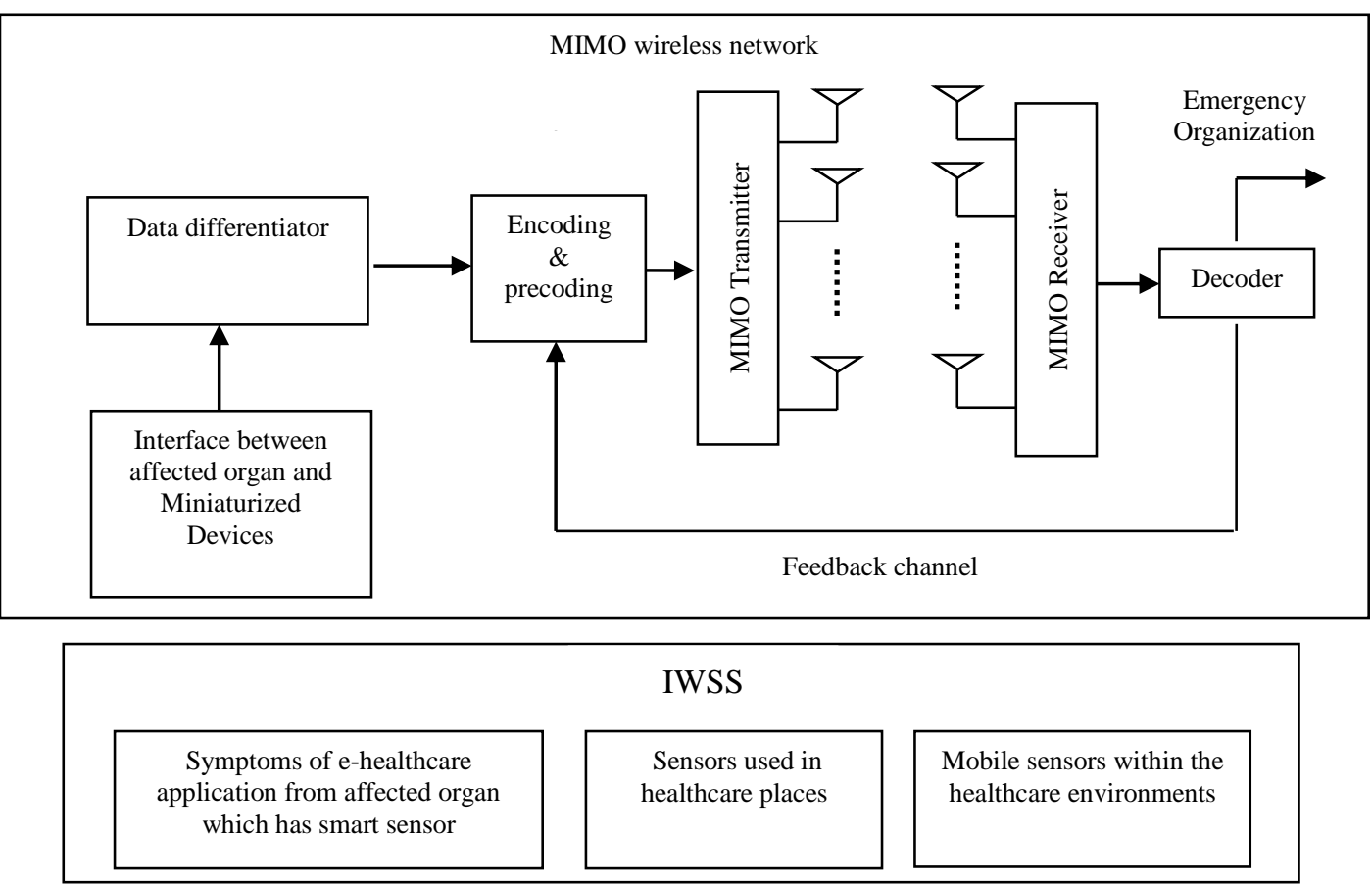

Fig. 2. Theoretical model of healthcare system based on MIMO.

In Fig. 2, application part contains all the e-healthcare applications. Then we use the infrastructure of the interfacing unit which is responsible for transferring data from MIMO network to healthcare unit where all applications are focused. Finally, all sensors in IWSS collect appropriate information from the patients and send through the MIMO network which passes the messages to applications and emergencies according to the situations. In order to verify our proposed model, we have considered glucose monitoring of diabetes as an example. In this model, a non-linear mathematical approach, which is the Pn-manifold, plays an important role in improving the accuracy of glucose monitoring. The Pn-manifold considered in the MIMO communication channel have to be employed to make an efficient mobile IWSS could be used in all environments. An intuitive sense of patients and their disease and symptoms related to diabetes can be identified through the IWSS accurately and quickly. In all environments, the response time of each glucose level monitoring is very quick which helps to improve the e-healthcare.

\section{B. Method of Monitoring Glucose Levels}

Glucose sensors embedded with IWSS detect the start and end points of the blood vessel where start and end points are fixed (assume that fixed length is $3 \mathrm{~cm}$ ). Within these points, the same volume of blood samples for different situations such as blood before the meal, after the meal can be monitored. According to the theory of diabetic patient, when the glucose level is increased, the density of blood is also increased. Blood flowing through this vessel is time-dependent as well as water pump. Blood flowing rate depends on the glucose level, and other factors such as body, mass and index (BMI) and it is different in various situations. Glucose sensors will determine the blood type as well as monitor a glucose level of the diabetic patient without pricking the patient's body many times. Our intention is that diabetic patients should get a better solution through this model which will look after their body with their normal daily life.

As Einstein predicted particle in the living cell would behave just like a molecule in solution. The Brownian particle would diffuse according to a simple (1). Using this concept, displacement of the particle in molecular communication D is given as

$$
D=\sqrt{\left[\frac{k_{B} T \pi \eta R t}{6}\right]}
$$

Where, $k_{B}$ is the Boltzmann's constant and $T$ is the temperature of the molecular communication, $\eta$ is the 
viscosity of the liquid used in the e-healthcare application. In MCD development using molecular particles, $R$ is the size of the particle and $t$ is time. In this theoretical model, minimum energy is also important when MCD is designed. Using (1), scientists know that molecules' kinetic energy $\left(k_{B} T / 2\right)$ depends on temperature. Sensors collect the average energy of the diabetic in various points.

Albert Einstein's famous formula $\mathrm{E}=\mathrm{mC}^{2}$ can be used as a theory which formulates a theoretical model of measuring the glucose level in the blood. Here, E, m, C are energy, mass, the speed of the light respectively. In blood vessels, volume (v) of pure blood can be measured using $m=d v$, where $d$ is the density of pure blood. If blood has sugar, the density of blood will be high. So mass will be increased, and energy will be calculated using $\mathrm{E}=\mathrm{dvC}^{2}$. Using light wave and IWSS, which measures the energy levels, it will be very helpful to test the sugar level reading. If the energy level exceeds the threshold limit, we can say that sugar level is high. Otherwise, the glucose level is normal. Here, blood flow rate can also be considered with time-dependent data and information. As far as the MIMO operation and its non-linear functions are concerned, the proposed model can measure the e-healthcare problems efficiently and accurately. Here, complex channel matrix of MIMO system can be optimized through feedback and Pn-manifold of MIMO approach.

\section{Pn-manifold}

In this theoretical model, e-healthcare data from any application is sent through the MIMO channels and fed back to MIMO transmitter from the MIMO receiver. Here, feedback channel characterized by the Pn-manifold provides a better resolution which reduces the quantization noise through lowcomplex non-linear matrices. Further, Pn-manifold is used for optimizing quantization, which allows healthcare staffs to see the accurate test details of the patients. Basically, quality of the channels is dependant on the Pn-manifolds which determine the achievable rates of the channels.

Evidence of the Pn-manifold in communication systems studied in the literature review proved that accuracy of the channel performance based on the MIMO scheme was better than the existing approach when we employed the correct type of Pn-manifold. Therefore, our proposed model will allow us to improve the accuracy of the medical systems and e-healthcare applications.

\section{Results AND ANALYsis}

Accuracy depends on the efficient design which contains MIMO with Pn-manifold as a novel approach. In these results, we have considered proposed and conventional schemes in two different periods within 24 hours. Fig. 3 shows the glucose levels between the period of time 2:00 and 12:00. Despite many monitoring devices, the responding time of each test takes a long time in conventional techniques. As far as this responding time is concerned, the proposed model produces the results quickly and accurately.

Diabetes can take different types of medications according to the level of glucose, which depends on the food, activities, etc. As shown in Fig. 4, healthcare units have obtained some recordings of glucose concentrations from the diabetic after the lunch.

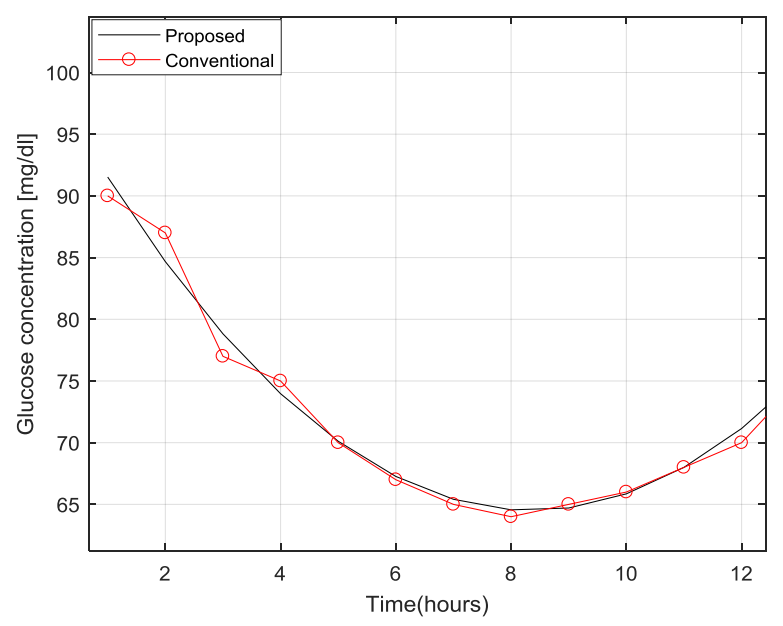

Fig. 3. Accuracy comparison during the daytime.

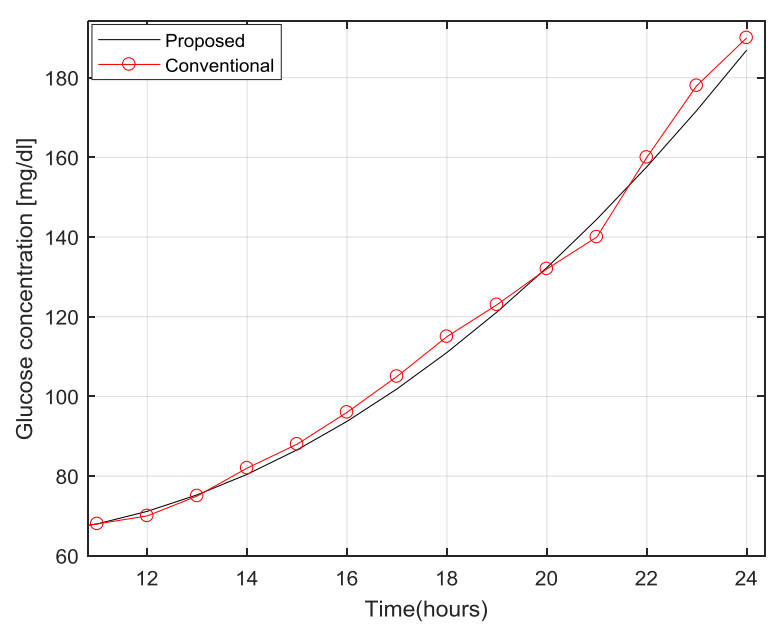

Fig. 4. Accuracy comparison during the evening and night.

In automation set with SCADA (supervisory control and data acquisition) in the e-healthcare, IWSS plays an important role in increasing network connections for multiple features network management such as Quality of service and reliability. Real-Time and secure wireless health monitoring is also part of the processing used in the e-healthcare system. Despite all facilities, monitoring devices such as blood pressure and heart rate monitor are considered as machines, which are motivating all the services used in the e-healthcare organization.

\section{CONCLUSIONS}

Through this theoretical model, we have studied the integration of the wearable smart sensors in medical communication and verified the accuracy as a performance of the selected e-healthcare application which is diabetes. Further, the proposed theoretical model can be employed to most of the e-healthcare applications in all environments and situations. Despite many healthcare facilities, IWSS with MIMO scheme provides maximum accuracy and better e-healthcare solutions 
to all diabetics. In order to improve the healthcare monitoring, the miniaturized device can be inserted into certain part of the body through the injection. Beside it is simple to use without many pricking pains per day, it reduces the overall cost and allows the patients and e-healthcare units/system to monitor the glucose level continuously.

Although the wearable jacket with many sensors provides dynamic monitoring facilities, mobile patients are still struggling to detect their health problems. Thus, IWSS detects all the symptoms accurately, quickly and efficiently. Hence, the diabetics take insulin tablets regularly to stimulate their metabolic rate. In this novel proposed approach, efficient communication is established through the MIMO system with Pn-manifold and feedback. In the future work, we can consider dynamic power management in e-healthcare [32] which allows researchers to minimize the overall energy and expenses in all e-healthcare applications. Here, adaptive packet size selection for IoT and smart sensors will play an important role in improving the e-healthcare services.

\section{ACKNOWLEDGMENT}

This work was funded by the Deanship of Scientific Research (DSR), King Abdulaziz University, Jeddah, under grant No. (D1435-701-611). The authors, therefore, acknowledge with thanks, DSR technical and financial support.

\section{REFERENCES}

[1] Baig, Mirza Mansoor, Hamid Gholamhosseini, Aasia A. Moqeem, Farhaan Mirza, and Maria Lindén. "A systematic review of wearable patient monitoring systems-current challenges and opportunities for clinical adoption." J. Med. Syst. 41, no. 7 (2017): 115.

[2] Wang, Stephen, Yilin Ji, David Gibbins, and Xuefeng Yin. "Impact of dynamic wideband MIMO body channel characteristics on healthcare rehabilitation of walking." IEEE Antennas and Wireless Propagation Letters 16 (2017): 505-508.

[3] Taleb, Tarik, Dario Bottazzi, Mohsen Guizani, and Hammadi NaitCharif. "ANGELAH: a framework for assisting elders at home." IEEE Journal on Selected Areas in Communications 27, no. 4 (2009): 480494.

[4] Peters, Anne L., Andrew J. Ahmann, Tadej Battelino, Alison Evert, Irl B. Hirsch, M. Hassan Murad, William E. Winter, and Howard Wolpert. "Diabetes Technology-Continuous Subcutaneous Insulin Infusion Therapy and Continuous Glucose Monitoring in Adults: An Endocrine Society Clinical Practice Guideline." The Journal of Clinical Endocrinology \& Metabolism 101, no. 11 (2016): 3922-3937.

[5] Lee, Hyunjae, Tae Kyu Choi, Young Bum Lee, Hye Rim Cho, Roozbeh Ghaffari, Liu Wang, Hyung Jin Choi et al. "A graphene-based electrochemical device with thermoresponsive microneedles for diabetes monitoring and therapy." Nature nanotechnology (2016).

[6] Levine, Jason C., Edith Burns, Jeffrey Whittle, Raymond Fleming, Paul Knudson, Steve Flax, and Howard Leventhal. "Randomized trial of technology-assisted self-monitoring of blood glucose by low-income seniors: improved glycemic control in type 2 diabetes mellitus." Journal of behavioral medicine 39, no. 6 (2016): 1001-1008.

[7] Kumpatla, S., \& Viswanathan, V. (2017). 'Diabetes and tuberculosis'-a co-epidemic of public health importance in the developing world. CURRENT SCIENCE, 113(7), 1296-1302.

[8] Hongasandra, N. R., More, P., Kumar, V., Jagannathan, A., \& Raghuram, N. (2017). The effectiveness of yoga for patients with diabetes mellitus. CURRENT SCIENCE, 113(7), 1337-1353.

[9] Krishnamachari R T, Varanasi M K.(2014), MIMO systems with quantized covariance feedback. IEEE Transactions on Signal Processing; 62: 485-495.
[10] Ma, Yujun, Yulei Wang, Jun Yang, Yiming Miao, and Wei Li. "Big Health Application System based on Health Internet of Things and Big Data." IEEE Access (2016).

[11] Y.-J. Ma, Y. Zhang, O. M. Dung, R. Li, and D.-Q. Zhang, "Health internet of things: recent applications and outlook," Journal of Internet Technology, vol. 16, no. 2, pp. 351-362, 2015.

[12] M. Chen, Y. Ma, Y. Li, Y. Zhang, and C.-H. Youn, "Wearable 2.0: Enable human-cloud integration in next generation healthcare system," IEEE Communications Magazine, vol. 54, no. 11, 2016.

[13] Aguirre, E., Lopez-Iturri, P., Azpilicueta, L., Rivarés, C., Astrain, J.J., Villadangos, J. and Falcone, F., 2016. Design and performance analysis of wireless body area networks in complex indoor e-Health hospital environments for remote patient monitoring. International Journal of Distributed Sensor Networks, 12(9), p.1550147716668063

[14] Balanou, Evangelia, Mark van Gils, and Toni Vanhala. "State-of-the-art of wearable EEG for personalized health applications." In PHealth 2013: Proceedings of the 10th International Conference on Wearable Micro and Nano Technologies for Personalized Health, vol. 189, p. 119-124. IOS Press, 2013

[15] Toschi, E., and Wolpert, H., Utility of Continuous Glucose Monitoring in Type 1 and Type 2 Diabetes. Endocrinology and metabolism clinics of North America, 45(4), 2016, pp.895-904.

[16] Dementyev, Artem, and Joshua R. Smith. "A wearable UHF RFIDbased EEG system." In RFID (RFID), 2013 IEEE International Conference on, pp. 1-7. IEEE, 2013.

[17] Jung, Sang-Joong, Risto Myllylä, and Wan-Young Chung. "Wireless machine-to-machine healthcare solution using android mobile devices in global networks." IEEE Sensors Journal 13, no. 5 (2013): 1419-1424.

[18] Lin, C-T., L-W. Ko, M-H. Chang, J-R. Duann, J-Y. Chen, T-P. Su, and T-P. Jung. "Review of wireless and wearable electroencephalogram systems and brain-computer interfaces-a mini-review." Gerontology 56, no. 1 (2009): 112-119.

[19] Dagtas, S., Pekhteryev, G., Sahinoglu, Z., Cam, H., \& Challa, N. (2008). Real-Time and Secure Wireless HealthMonitoring. USA: Hindawi Publishing Corporation, International Journal of Telemedicine and Applications.

[20] Balanou, Evangelia, Mark van Gils, and Toni Vanhala. "State-of-the-art of wearable EEG for personalized health applications." In PHealth 2013: Proceedings of the 10th International Conference on Wearable Micro and Nano Technologies for Personalized Health, vol. 189, p. 119-124. IOS Press, 2013.

[21] Shyamkumar, Prashanth, Sechang Oh, Nilanjan Banerjee, and Vijay K. Varadan. "A wearable remote brain machine interface using smartphones and the mobile network." In Advances in Science and Technology, vol. 85, pp. 11-16. Trans Tech Publications, 2013.

[22] Qureshi, Muhammad Shuaib, and Vijey Thayananthan. "Self-Optimized Routing Algorithm for mHealth and Remote Health Monitoring." Journal of Medical Imaging and Health Informatics 6, no. 1 (2016): 189193.

[23] Joonhyung Kim, Donghyuk Han, Jong-Moon Chung, (2010), "Virtual MIMO Based Wireless Communication for Remote Medical Condition Monitoring," Aging Friendly Technology for Health and Independence Lecture Notes in Computer Science Volume 6159, pp 259-262.

[24] Lal, Kumari Nidhi, and Anoj Kumar. "E-health application over 5G using Content-Centric networking (CCN)." In IoT and Application (ICIOT), 2017 International Conference on, pp. 1-5. IEEE, 2017.

[25] Hu, Fei, Yang Xiao, and Qi Hao. "Congestion-aware, loss-resilient biomonitoring sensor networking for mobile health applications." IEEE Journal on Selected Areas in Communications 27, no. 4 (2009): 450465.

[26] Nihtianov, Stoyan, Zhichao Tan, and Boby George. "New Trends in Smart Sensors for Industrial Applications-Part I." IEEE Transactions on Industrial Electronics 64, no. 9 (2017): 7281-7283.

[27] Tadashi Nakano, Andrew W. Eckford, and Tokuko Haraguchi, (2013) "Molecular Communication," Cambridge University Press, United Kingdom, ISBN 978-1-107-02308-6 (hardback).

[28] Yapici, Murat Kaya, and Tamador Elboshra Alkhidir. "Intelligent Medical Garments with Graphene-Functionalized Smart-Cloth ECG Sensors." Sensors 17, no. 4 (2017): 875. 
[29] Colomer-Farrarons, J., Miribel-Català, P., Samitier, J., Arundell, M., \& Rodrsguez, I. (2009). Design of a miniaturized electrochemical instrument for in- situ O2 monitoring. (Proceedings Paper), VLSI Circuits and Systems IV, Microtechnologies for the NewMillennium, Vol. 7363, Spie'09.

[30] Ouyang, Yuehui, David J. Love, and William J. Chappell. "Body-worn distributed MIMO system." IEEE Transactions on Vehicular Technology 58, no. 4 (2009): 1752-1765.
[31] M. Jayasheela and A. Rajeswari, (2012), "Improved Successive Interference Cancellation for MIMO/UWB-Based Wireless Body Area Network," International Journal of Antennas and Propagation Volume 2012.

[32] Chen, Xi, Ming Ma, and Anfeng Liu. "Dynamic power management and adaptive packet size selection for IoT in e-healthcare." Computers \& Electrical Engineering (2017). 